\title{
Branched chain amino acids: Passive biomarkers or the key to the pathogenesis of cardiometabolic diseases?
}

\author{
Marta Siomkajto ${ }^{\mathrm{A}, \mathrm{D}}$, Jacek Daroszewski ${ }^{\mathrm{A}, \mathrm{E}, \mathrm{F}}$ \\ Department and Clinic of Endocrinology, Diabetes and Isotope Therapy, Wroclaw Medical University, Poland \\ A - research concept and design; B - collection and/or assembly of data; $C$ - data analysis and interpretation; \\ $D$ - writing the article; $E$ - critical revision of the article; $F$ - final approval of the article
}

Address for correspondence

Marta Siomkajło

E-mail: m-s@10g.pl

Funding sources

None declared

Conflict of interest

None declared

Received on July 11, 2018

Reviewed on August 23, 2018

Accepted on February 18, 2019

Published online on August 19, 2019

\begin{abstract}
The metabolomic approach to research on lifestyle diseases has led to the discovery of new potential biomarkers of pathological conditions as well as key metabolic pathways that may become targets of therapeutic intervention. Current evidence supports plasma branched chain amino acids (BCAAs) as potential diagnostic and prognostic biomarkers of cardiometabolic diseases. However, the biological mechanisms of the associations that have been identified are still not completely understood and should be clarified before implementing BCAA-based biomarkers in the clinical setting. The most crucial issue that needs to be solved first is determining whether BCAA plasma profile disturbances are only passive biomarkers or whether they facilitate dysmetabolic processes. In this context, further research is also warranted to investigate the role of dietary BCAAs. Gaining this knowledge would be significant progress in molecular nutrition research, providing perspective for target therapeutic and prophylactic interventions. This paper provides a comprehensive review of the main hypotheses and mechanistic models that consider circulating BCAAs both as passive biomarkers and as contributors to cardiometabolic diseases.
\end{abstract}

Key words: amino acids, biomarker, metabolomics, BCAA, cardiometabolic diseases

Cite as

Siomkajło M, Daroszewski J. Branched chain amino acids: Passive biomarkers or the key to the pathogenesis of cardiometabolic diseases? Adv Clin Exp Med. 2019;28(9):1263-1269. doi:10.17219/acem/104542

DOI

10.17219/acem/104542

\section{Copyright}

Copyright by Author(s)

This is an article distributed under the terms of the

Creative Commons Attribution Non-Commercial License

(http://creativecommons.org/licenses/by-nc-nd/4.0/) 
The metabolomic approach to research on lifestyle diseases has led to the discovery of new potential biomarkers of pathological conditions as well as key metabolic pathways that may become targets of therapeutic interventions. ${ }^{1}$ Evidence from recent studies indicates that there is a close association between the plasma concentrations of branched chain amino acids (BCAAs) and cardiometabolic diseases (CMDs) such as diabetes (DM2), insulin resistance (IR), cardiovascular disease (CVD), obesity, and metabolic syndrome (MS). ${ }^{2}$ Moreover, it has been shown that BCAAs have diagnostic and prognostic value, correlate with positive outcomes of therapeutic interventions, and can be used to differentiate metabolically healthy from metabolically unhealthy obese patients. Branched chain amino acids have therefore been proposed as valuable diagnostic and prognostic biomarkers with considerable clinical potential. ${ }^{2,3}$

Branched chain amino acids comprise 3 essential amino acids (AA): leucine (Leu), isoleucine (Ile) and valine (Val), which account for $35-40 \%$ of the indispensable dietary amino acids in body protein and $14 \%$ of the total amino acids in skeletal muscle. They share common membrane transport systems and enzymes for their transamination and irreversible oxidation. ${ }^{4}$ Branched chain amino acids are not only sources of energy and passive substrates for synthesis, but are also very important as signaling molecules and cell regulators. They influence glucose, protein and lipid metabolism. Changes in amino acid availability have profound effects on cell signaling, gene expression, brain, and neuroendocrine functions. ${ }^{5}$

The mechanisms underlying the relationship of plasma BCAAs to diseases processes are still not completely understood and need to be clarified prior to the use of BCAAs as biomarkers in clinical practice. ${ }^{3}$ The most crucial issue that need s to be solved first is determining whether BCAA plasma profile disturbances are only passive biomarkers or whether they facilitate dysmetabolic processes; this has not yet been determined. ${ }^{6}$ Paradoxically, there is a great deal of evidence showing the benefits of BCAA supplementation and diets high in BCAA-rich proteins. ${ }^{7}$ The reasons for these seemingly contradictory observations, however, remain elusive.

Branched chain amino acid catabolism impairment in 1 or more tissues, changes in cellular uptake from the blood, increases in proteolysis, IR or excessive intake have been identified as potential causes of elevated plasma BCAA concentration. It has been suggested that BCAAs may have a causative role in CMD development, mainly through nutrient-sensitive factor overstimulation and IR promotion, ${ }^{8}$ but also by direct and indirect influence on brain function. ${ }^{9,10}$ The association of BCAAs with coronary artery disease seems to be independent of IR and diabetes. ${ }^{11}$ There are suggestions that some disturbances of BCAA metabolism could be part of an early, yet to be elucidated, metabolic change that precedes CMD development, and links DM2 and CVD pathogenesis. ${ }^{12}$ Some of the essential issues regarding BCAA metabolism in the context of CMD pathogenesis are highlighted in this review.

\section{BCAAs as passive CMD biomarkers}

\section{Excessive intake}

Considering that BCAAs are essential AAs, which means that they cannot be synthesized in the organism de novo, plasma BCAA concentration depends on the intensity of their utilization, protein turnover and the amount of dietary supply. ${ }^{6}$

The robust regulation and capacity of the BCAA catabolic pathway makes increased intake a highly doubtful cause of elevated plasma BCAA levels. In the postprandial state the BCAA metabolism rate increases with supply and the plasma concentration remains within a narrow range. The capacity of BCAA catabolism reaches a plateau and the plasma BCAA concentration increases only when intake greatly exceeds the normal daily supply. ${ }^{13}$ Studies showing an association between CMDs and plasma BCAA concentration have mostly been performed in prandial situations. Previous studies have showed no association between prandial plasma BCAA concentration and dietary intake. Within $5 \mathrm{~h}$ of a meal the plasma BCAA concentration should bounce back to the baseline. ${ }^{14}$ However, excessive intake should be considered a meaningful trigger in the situation of BCAA catabolic pathway disruption.

\section{Impairment of BCAA catabolism}

The metabolism of BCAAs has 3 stages (transamination, oxidative decarboxylation and oxidation), is very effective and is strictly regulated to avoid toxic excess. ${ }^{16}$ The first, reversible step takes place in peripheral tissues and is catabolized by the mitochondrial isoform of branched-chain aminotransferase (BCATm, encoded by BCAT2). The second, highly regulated, irreversible step, which is critical for BCAA catabolism, is catalyzed by the multienzyme mitochondrial branched-chain-ketoacid dehydrogenase complex (BCKDC). The BCKDC irreversibly oxidizes BCAAs to their respective ketoacids. The ketoacids formed are further metabolized by multiple enzymatic steps within the mitochondrial-matrix, eventually forming lipogenic, ketogenic or glucogenic substrates (acetoacetyl-CoA, acetyl-CoA and propionyl-CoA). ${ }^{17}$

In humans BCAA metabolism takes place mainly in muscles and adipose tissue. ${ }^{18}$ Studies on rodents indicate that BCATm and BCKDC in adipose tissue have direct and significant influence on plasma BCAA concentration. ${ }^{19}$

The expression and activity of BCKDC can be altered by numerous metabolic factors. The overall enzymatic activity is controlled by the phosphorylation (inactivation)/ dephosphorylation (activation) cycle. Intense physical activity, high abundance of branched-chain ketoacids (from 
a high-protein diet, BCAA supplementation, starvation, etc.), glikokortykosteroids or clofibrate cause reductions in kinase activity. Insulin, free fatty acids (FFAs), a lowprotein diet, hyperthyroidism, and $17 \beta$-estradiol enhance kinase activity. ${ }^{20}$

Visceral adipose tissue in metabolically unhealthy obese people and rodents is characterized by reduced expression of BCKDC enzymes. It has been suggested that insulin action or a healthy metabolic/inflammatory phenotype is responsible for the modulation of BCAA catabolic enzyme expression. ${ }^{21}$ Morbidly obese women with MS have been found to have reduced BCAA catabolic enzyme expression compared to equally obese but metabolically healthy controls. ${ }^{21}$ Treatment with PPAR agonists has been shown to lead to increases in BCAA catabolic pathway transcripts. ${ }^{22}$ Bariatric surgery in morbidly obese patients leads to reductions in plasma BCAA concentration of approx. $35 \%$ and concomitant increases in $\mathrm{mBCAT}$ and BCKDC in omental and subcutaneous tissue. Moreover, decreases in plasma BCAA concentration predict metabolic improvement irrespective of body weight reduction. ${ }^{23}$

Manipulation of GLUT4 transporter expression in adipose tissue leads to a selective decrease in BCAA catabolic enzyme activity with a concomitant increase in plasma BCAA concentration. Both insulin and FFAs (both of which are increased in IR) are negative regulators of the BCKDC. ${ }^{24}$ It is suspected that diminished muscle uptake of glucose with increased glucose availability and corresponding hyperinsulinemia lead to selective adipose tissue BCAA catabolism impairment and increase BCAA plasma concentration. This suggests that IR and obesity may cause impairment of BCAA degradation in adipose tissue. ${ }^{18}$

Genetic variations in the expression of the genes encoding key BCAA catabolic enzymes or the proteins that control protein synthesis and turnover may be considered further contributors to BCAA dysmetabolism. The BCKDC encoding gene has been identified as one of the major genes associated with DM2 and obesity-related metabolic dysfunction. ${ }^{25}$ Studies on monozygotic twins indicate that BCAA metabolic pathway gene expression undergoes functional negative regulation in obese individuals, which leads to elevated plasma BCAA concentration. ${ }^{18}$ Positive functional regulation of BCAA catabolic pathway gene expression with concomitant lower plasma BCAA concentration seems to be associated with higher levels of physical activity. ${ }^{26}$

It has been demonstrated that brain insulin plays a significant role in BCAA metabolism through positive regulation of hepatic BCKDC. It is suspected that hypothalamic IR is responsible for disruption of hepatic BCAA catabolism, and persistently increased BCAA concentrations can exacerbate hypothalamic IR by mTOR hyperstymulation, starting a vicious cycle. ${ }^{27}$

Taking all of the above together: BCAA catabolic pathway is dampened in obesity-related metabolic disorders.
Regulation mainly involves the BCKDC in adipose tissue and takes place on several levels: direct enzyme regulation (activators, inhibitors, allosteric factors), post-translational modifications and gene expression. Insulin resistance, adipocyte dysfunction and physical activity have been identified as the most important factors influencing the BCAA catabolic pathway. Impairment of BCAA catabolism is functional and can be improved by interventions increasing insulin sensitivity.

\section{Increased protein turnover}

Most studies indicating a relationship between BCAAs and CMDs have been performed in the fasting state, when protein anabolism is minimized; differences in protein synthesis rates are therefore unlikely to be a meaningful factor driving plasma BCAA differences. Similarly, increased protein and fat-free mass catabolism in IR and reasonably controlled DM2 should not be considered important in plasma BCAA differences, because they do not explain the selectivity of hyperaminoacydemia. ${ }^{17}$

\section{Gut microbiota}

Gut microbiota are involved in the metabolism of several amino acids and have been shown to be important factors in the supply of BCAAs to mammalian hosts ${ }^{28}$; at the same time, BCAAs participate in bacterial metabolism and are considered important regulators of intestinal microbial species and diversity. ${ }^{5}$ Disruption of gut microbial composition and function (dysbiosis) is implicated in the pathogenesis of CMDs such as obesity, IR, MS, and DM2 ${ }^{29,30}$ It is conceivable that the altered composition of gut microbiota that is observed in DM2 and obese patients modulates the plasma BCAA profile. ${ }^{28,31}$ This may also go some way to explaining why dietary protein content does not necessarily reflect changes in the plasma AA profile. The association between dysbiosis, plasma AA profile changes and CMDs needs further evaluation.

\section{BCAAs as contributors to CMDs}

\section{mTOR overstimulation}

Branched chain amino acids are very important signaling molecules and cell regulators that influence many key cell signaling pathways. ${ }^{32}$ Some AA transporters also play the role of receptors and conduct information about the nutritional state, the quantity and quality of extra and intracellular AAs to nutrient-sensitive factors such as the general control nonderepressible 2 (GCN2) kinase and the mammalian target of rapamycin complex (mTORC). ${ }^{33}$ However, sensing pathways are also affected 
in negative ways. Under certain conditions BCAAs are considered IR-promoting factors through overstimulation of the adenosine monophosphate activated protein kinase (AMPK), mTOR and GCN2. ${ }^{34}$

mTORC1 is a nutrient-sensitive protein complex that promotes the synthesis of proteins and lipids, the growth, proliferation and differentiation of cells, and the biogenesis of mitochondria, while it inhibits autophagy. ${ }^{35}$ The activity of the complex is regulated by pathways related to insulin and growth factors, and is independently stimulated by cell energy status, glucose and AA availability. Thus, mTORC1 integrates extra and intracellular signals to maintain balance in the organism's energy. ${ }^{35}$

Insulin and insulin growth factor (IGF) stimulate mTOR via the IRS/PI3K/Akt pathway and activation of the mTORC1 inducer GTPase Rheb. Rheb is regulated by the cell nutrient sensor AMPK. In states of high energy abundance, the ATP/cAMP ratio is high, repressive function of AMPK on Rheb is reduced and mTORC1 is activated. ${ }^{36}$ It has been shown that AAs, particularly Leu, play a critical role in mTORC1 activation by enabling translocation of inactive mTORC1 complex to cell compartments with a high abundance of Rheb protein. ${ }^{37}$ Moreover, BCAAs influence mTOR activity both directly and by inactivating other mTOR repressors, like GCN2. GCN2 inhibition also reduces repression of sterol regulatory element-binding protein 1 (SREBP-1), promoting lipogenesis. ${ }^{8}$

A negative feedback signal emanating from the mTOR pathway end-product - active p70 S6K - results in serine phosphorylation of insulin receptor substrate (IRS) and thus is involved in negative regulation of insulin signaling. Further, mTOC1 overstimulation by a high abundance of nutrients, proinflammatory cytokines and extra cell stimulation by insulin and IGF will result in IR on the IRS level, with concomitant lipid synthesis promotion via SREBP $-1 .^{37}$ This is the foundation for the hypothesis that an energy-dense diet rich in AAs coming from dairy (highinsulinogenic, with the potential to evoke IGF action) will lead to IR via mTOR overstimulation. ${ }^{37}$ Furthermore, taking into account the previous considerations, it should be noted that the effect could be exacerbated by impairment of BCAA catabolism.

\section{Brain function and the neurobiological origin of obesity and metabolic diseases}

The central nervous system (CNS), particularly the hypothalamus, plays an important role in the regulation of food intake, energy balance and glucose homeostasis. ${ }^{38}$ It has been suggested that disruptions in the brain signaling systems play an important role in the pathogenesis of DM2 and metabolic syndrome. ${ }^{39,40}$

Branched chain amino acids have both direct and indirect influence on brain neurochemistry. They compete for blood-brain barrier carriers with the precursors for neurotransmitters synthesis: aromatic amino acids
(AAAs). Thus, the plasma AA pattern determines AA supply to the brain and consequently also determines neurotransmission. ${ }^{41}$

It has been posited that addiction and obesity have the same neurobiological origin and are based on serotonergic and dopaminergic transmission that regulates the neuron systems of reward, control, motivation, and conditioning. ${ }^{42}$ Modulation of the proportions of BCAAs and AAAs leads to changes in serotonergic and dopaminergic transmission. ${ }^{42}$

The dopaminergic pathway, which relies on tyrosine (Tyr) and phenylalanine (Phe) supply, is involved in food-based rewards and is closely linked to hunger. ${ }^{43}$ Serotonergic neurotransmission, which is affected by tryptophan (Trp) availability, is essential for the regulation of reward-related behaviors and is involved in the regulation of food intake, body weight, mood, and autonomic functions. ${ }^{39,40}$ Receptor- and brain area-specific disruptions of serotonin signaling lead to hyperphagia, disturbed energy expenditure, obesity and reduced insulin sensitivity. ${ }^{39}$ "Carbohydrate craving" behavior, which is considered a driver of obesity, restores serotonergic neurotransmission by increasing tryptophan's flux across the blood-brain barrier. ${ }^{44}$ The appetite suppressant effects of fenfluramine and dexfenfluramine, which were formerly effectively used for obesity treatment, were based on increments in serotonin transmission. ${ }^{45}$ Central administration of serotonin-depleting agents in rats has been shown to result in hyperphagia and increased body weight. ${ }^{46}$ Some studies have suggested an association between reduced central serotonergic responsivity and MS. ${ }^{40}$

Insulin lowers plasma concentrations of BCAAs, Tyr and Phe. Tryptophan circulates in the blood mostly bound to albumin, so plasma Trp concentrations are not as strongly affected by insulin action. ${ }^{41}$ Ingestion of carbohydrates increases the plasma ratio of tryptophan to other large neutral amino acids, leading to significantly increased serotonin synthesis and release. In contrast, ingestion of a protein-containing meal is usually reported to lower serum Trp concentrations in relation to competitors, to reduce brain Trp uptake and levels, and to diminish serotonin synthesis and release. ${ }^{47}$ Based on this, we can assume that in IR, Trp uptake in the brain would be diminished, disrupting serotonin transmission in the complex neurobehavioral circuit that controls appetite. To benefit from higher serotonin release, exhibit positive mood changes and reach satiety, one must provide food with a much higher glycemic load, producing higher insulin release.

Leu acts on the CNS both directly, by activating the mTOR pathway, and indirectly, through its metabolites, and exerts both positive and negative effects on whole body metabolism. It has been linked to nutrient-sensitive hypothalamic neurons that affect the behavioral and physiological determinants of energy balance and are associated with the pathophysiology of obesity and metabolic diseases. ${ }^{9}$

Central Leu infusion decreases food intake and body 
weight. ${ }^{9,48}$ However, in most studies no central anorexogenic effect of clinical importance was observed when Leu was provided orally. The rationale for this discrepancy could be explained by the fact that increases in plasma Leu do not necessary reflect significant increments in the CNS. Direct Leu infusion into the CNS does not reproduce physiological conditions; however, it is conceivable that Leu has a central anorexogenic role in appetite regulation and obesity. ${ }^{48}$

Hypothalamic BCAA metabolism is involved in central glucoregulation through the brain-liver circuit and the evoked effects appear to oppose those that are exerted on the periphery. ${ }^{49}$ The Leu metabolite malonyl-CoA, which is further utilized to oleoyl-CoA, is directly responsible for the glucoregulatory effects. Activation of hypothalamic $K_{\text {ATP }}$ channels generates a neurogenic signal that is relayed to the liver via the hepatic branch of the vagus nerve to reduce hepatic glucose production through a reduction in gluconeogenesis and glycogenolysis. ${ }^{50}$ This model of central glucose regulation has been confirmed by studies using both central and systemic BCAA administration. ${ }^{49,50}$ It has been shown that factors that disrupt central Leu-sensing can contribute to glycemic dysregulation and hyperglycemia. ${ }^{49}$ Central glucoregulation can be also attenuated by diet. Animal studies have shown, for example, that diets high in saturated fat blunt the mediobasal hypothalamus response to Leu. ${ }^{49,51}$

Leu central signaling can also exert negative metabolic effects by inducing peripheral IR in chronic BCAA elevation, leading to persistent hypothalamic mTOR overstimulation. ${ }^{27}$ The ambiguous effects of Leu can be explained by the dualistic mTOR/insulin sensitivity model, which assumes that the association of mTOR activity and insulin sensitivity seems to follow a U-shaped curve, where mTOR activity that is either too low or too high has a negative metabolic effect. ${ }^{36}$

\section{The detrimental effects of excessive BCAA catabolic flux}

Some studies suggest that BCAA catabolism rather than BCAA concentration is associated with metabolic dysfunction. ${ }^{52,53}$ Newgard et al. observed that IR is associated not only with BCAAs but also with BCAA catabolites, indicating enhanced BCAA catabolism. They hypothesized that enhanced BCAA catabolism may reflect overfeeding, particularly an overload of the BCAA catabolic pathway in obese subjects. ${ }^{54}$ Experimental studies with rats showed that enhanced BCAA flux synergizes with hyperlipidemia, making a contribution to IR. The disruption was accompanied by chronic mTOR activation, phosphorylation of IRS1 (ser307) in skeletal muscles and accumulation of incompletely oxidized substrates, leading to mitochondrial stress. ${ }^{37}$

Another explanation for how excess catabolic flux of BCAAs can lead to IR involves a catabolic intermediate of valine - 3-hydroxyisobutyrate (3-HIB) - the paracrine metabolite that regulates the trans-endothelial flux of FFAs. It is suspected that excessive secretion of 3-HIB from muscles leads to excess trans-endothelial FFA import into muscles, accumulation of lipotoxic, incompletely esterified intermediates, and blunted insulin signaling. ${ }^{53}$ Moreover, increased plasma levels of 3-HIB were found to be a marker of future DM2 development. ${ }^{55}$

\section{Does dietary BCAA matter?}

There are multiple reports about the influence of BCAArich diets on metabolic health, but the conclusions are inconsistent. ${ }^{24}$ Some of them indicate detrimental effects, such as the promotion of IR, metabolic dysfunctions and DM2. ${ }^{56,57}$ Others suggest beneficial outcomes, including a decreased risk of DM2, ${ }^{7,58}$ which, in the light of the above considerations, may seem paradoxical. However, it should be mentioned that both Leu and Ile have the capacity to increase glucose clearance by different modes of action: by the promotion of insulin secretion (Leu) as well as in insulin- and mTOR-independent mechanisms: by increasing cellular uptake (Leu and Ile), by promoting glycogenogenesis (Leu) and by downregulating gluconeogenesis (Ile). ${ }^{59}$ It seems that the influence of dietary BCAA on glucose metabolism and insulin signaling are sophisticated, and that the final outcome depends on the context in which BCAAs are administered, such as actual insulin sensitivity, the overall energy balance, the dosage, the site of action, the duration, background lipid profiles, other macronutrients in the diet, and muscle mass. ${ }^{60}$ Diet intervention studies have many limitations and inter-individual differences in the metabolomic effects of dietary interventions have been reported. ${ }^{61}$ Certain individuals display greater stability in their metabolic phenotype. ${ }^{61}$ The individual microbiome ${ }^{28}$ and the potential of high-protein diets to increase satiety through the incretin-CNS axis should also be considered possible confounding factors. ${ }^{58}$

\section{Conclusions}

Metabolomics research has identified plasma BCAA as a potentially valuable biomarker of CMD. However, despite numerous hypotheses, the mechanisms underlying the relationship of plasma BCAA to the disease process have still not been clarified. Determination of the place and role of plasma and BCAA diet in the pathogenesis of CMDs should be made prior to implementing BCAA-based biomarkers in clinical settings, and could provide a perspective for target therapeutic and prophylactic interventions.

The complexity of BCAA interactions with metabolic pathways, along with the multitude of diet-delivered and internal factors that influence them, constitutes a great challenge for molecular nutrition research, personalized nutrition and medicine. 


\section{References}

1. Collino S, Martin FPJ. Rezzi S. Clinical metabolomics paves the way towards future healthcare strategies. Br J Clin Pharmacol. 2013;75(3): 619-629.

2. Batch BC, Hyland K, Svetkey LP. Branch chain amino acids: Biomarkers of health and disease. Curr Opin Clin NutrMetab Care. 2014;17(1):86-89.

3. Siomkajło M, Rybka J, Mierzchała-Pasierb M, et al. Specific plasma amino acid disturbances associated with metabolic syndrome. Endocrine. 2017;58(3):553-562.

4. Manoli I, Venditti CP. Disorders of branched chain amino acid metabolism. Trans/ Sci Rare Dis. 2016;7;1(2):91-110.

5. Nie C, He T, Zhang W, Zhang G, Ma X. Branched chain amino acids: Beyond nutrition metabolism. Int J Mol Sci. 2018;19(4):954.

6. Yoon MS. The emerging role of branched-chain amino acids in insulin resistance and metabolism. Nutrients. 2016;8(7):405.

7. Layman DK, Walker DA. Potential importance of leucine in treatment of obesity and the metabolic syndrome. J Nutr. 2006;136(1 Suppl): 319-323.

8. Lu J, Xie G, Jia W. Insulin resistance and the metabolism of branchedchain amino acids. Front Med. 2013;7(1):53-59.

9. Blouet C, Jo YH, Li X, Schwartz GJ. Mediobasal hypothalamic leucine sensing regulates food intake through activation of a hypothalamusbrainstem circuit. J Neurosci. 2009;29(26):8302-8311.

10. Fernstrom JD. Large neutral amino acids: Dietary effects on brain neurochemistry and function. Amino Acids. 2013;45(3):419-430.

11. Bhattacharya S, Granger CB, Craig D, et al. Validation of the association between a branched chain amino acid metabolite profile and extremes of coronary artery disease in patients referred for cardiac catheterization. Atherosclerosis. 2014;232(1):191-196.

12. Magnusson $M$, Lewis GD, Ericson $U$, et al. A diabetes-predictive amino acid score and future cardiovascular disease. Eur Heart J. 2013;34(26): 1982-1989.

13. Elango R, Ball RO, Pencharz PB. Tolerability of Leucine in Humans. Branched Chain Amino Acids in Clinical Nutrition. $2^{\text {nd }}$ ed. New York, NY: Springer Science\&Business Media; 2015

14. Noguchi Y, Zhang QW, Sugimoto T, et al. Network analysis of plas$\mathrm{ma}$ and tissue amino acids and the generation of an amino index for potential diagnostic use. Am J Clin Nutr. 2006;83(2):513-519.

15. Tai ES, Tan MLS, Stevens RD, et al. Insulin resistance is associated with a metabolic profile of altered protein metabolism in Chinese and Asian-Indian men. Diabetologia. 2010;53(4):757-767.

16. Tom A, Nair KS. Assessment of branched-chain amino acid status and potential for biomarkers. J Nutr. 2006;136(1 Suppl):324S-330S.

17. Adams $\mathrm{SH}$. Emerging perspectives on essential amino acid metabolism in obesity and the insulin-resistant state. Adv Nutr Bethesda Md 2011;2(6):445-456

18. Laferrère $B$, Pietiläinen $K$, Boirie $Y$. Weight Loss and branched chain amino acids and their metabolites. In: Rajendram R, Preedy VR, Patel VB, eds. Branched Chain Amino Acids in Clinical Nutrition: Volume2. New York, NY: Humana Press, Springer Science+Business Media; 2015:251-262.

19. Joshi MA, Jeoung $\mathrm{NH}$, Obayashi $\mathrm{M}$, et al. Impaired growth and neurological abnormalities in branched-chain alpha-keto acid dehydrogenase kinase-deficient mice. Biochem J. 2006;400(1):153-162.

20. Shimomura Y, Obayashi M, Murakami T, Harris RA. Regulation of branched-chain amino acid catabolism: Nutritional and hormonal regulation of activity and expression of the branched-chain alphaketo acid dehydrogenase kinase. Curr Opin Clin Nutr Metab Care. 2001; 4(5):419-423.

21. Lackey DE, Lynch CJ, Olson KC, et al. Regulation of adipose branchedchain amino acid catabolism enzyme expression and cross-adipose amino acid flux in human obesity. Am J Physiol Endocrinol Metab. 2013;304(11):1175-1187.

22. Sears DD, Hsiao G, Hsiao A, et al. Mechanisms of human insulin resistance and thiazolidinedione-mediated insulin sensitization. Proc Natl Acad Sci. 2009;106(44):18745-18750.

23. Laferrère $B$, Reilly $D$, Arias $S$, et al. Differential metabolic impact of gastric bypass surgery versus dietary intervention in obese diabetic subjects despite identical weight loss. Sci Trans/ Med. 2011;3(80):80re2.

24. Rietman A, Schwarz J, Tomé D, Kok FJ, Mensink M. High dietary protein intake, reducing or eliciting insulin resistance? Eur J Clin Nutr. 2014;68(9):973-979.
25. Tiffin N, Adie E, Turner F, et al. Computational disease gene identification: A concert of methods prioritizes type 2 diabetes and obesity candidate genes. Nucleic Acids Res. 2006;34(10):3067-3081.

26. Kujala UM, Mäkinen V-P, Heinonen I, et al. Long-term leisure-time physical activity and serum metabolome. Circulation. 2013;127(3):340-348.

27. Shin AC, Fasshauer $M$, Filatova $N$, et al. Brain insulin lowers circulating BCAA levels by inducing hepatic BCAA catabolism. Cell Metab. 2014;20(5):898-909.

28. Neis EPJG, Dejong CHC, Rensen SS. The role of microbial amino acid metabolism in host metabolism. Nutrients. 2015;7(4):2930-2946.

29. Festi D, Schiumerini R, Eusebi LH, Marasco G, Taddia M, Colecchia A. Gut microbiota and metabolic syndrome. World J Gastroenterol. 2014; 20(43):16079-16094.

30. Hartstra AV, Bouter KEC, Bäckhed F, Nieuwdorp M. Insights into the role of the microbiome in obesity and type 2 diabetes. Diabetes Care. 2015;38(1):159-165.

31. Pedersen HK, Gudmundsdottir V, Nielsen HB, et al. Human gut microbes impact host serum metabolome and insulin sensitivity. Nature. 2016; 535(7612):376-381.

32. Zhang S, Zeng X, Ren M, Mao X, Qiao S. Novel metabolic and physiological functions of branched chain amino acids: A review. J Anim Sci Biotechnol. 2017;8:10.

33. Hundal HS, Taylor PM. Amino acid transceptors: Gate keepers of nutrient exchange and regulators of nutrient signaling. Am J Physiol Endocrinol Metab. 2009:296(4):603-613.

34. Wang CX, Guo FF. Branched chain amino acids and metabolic regulation. Chin Sci Bull. 2013;1228-1235.

35. Catania C, Binder E, Cota D. mTORC1 signaling in energy balance and metabolic disease. Int J Obes. 2011;35(6):751-761.

36. Laplante M, Sabatini DM. mTOR signaling in growth control and disease. Cell. 2012;149(2):274-293.

37. Melnik BC. Leucine signaling in the pathogenesis of type 2 diabetes and obesity. World J Diabetes. 2012;3(3):38-53.

38. Morton GJ, Meek TH, Schwartz MW. Neurobiology of food intake in health and disease. Nat Rev Neurosci. 2014;15(6):367-378.

39. Shpakov AO, Derkach KV, Berstein LM. Brain signaling systems in the Type 2 diabetes and metabolic syndrome: Promising target to treat and prevent these diseases. Future Sci OA. 2015;1(3):FSO25.

40. Muldoon MF, Mackey RH, Williams KV, Korytkowski MT, Flory JD, Manuck SB. Low central nervous system serotonergic responsivity is associated with the metabolic syndrome and physical inactivity. J Clin Endocrinol Metab. 2004;89(1):266-271.

41. Cansev M, Wurtman RJ. 4 aromatic amino acids in the brain. In: Lajtha A, Oja SS, Schousboe A, Saransaari P, eds. Handbook of Neurochemistry \& Molecular Neurobiology. New York, NY: Springer US; 2007:59.

42. Singh M. Mood, food, and obesity. Front Psychol. 2014;5:925.

43. Carreiro AL, Dhillon J, Gordon S, et al. The macronutrients, appetite and energy intake. Annu Rev Nutr. 2016:36:73-103.

44. Wurtman RJ, Wurtman JJ. Brain serotonin, carbohydrate-craving, obesity and depression. Obes Res. 1995;3 Suppl 4:477-480.

45. Bello NT, Liang NC. The use of serotonergic drugs to treat obesity - is there any hope? Drug Des Devel Ther. 2011;5:95-109.

46. Breisch ST, Zemlan FP, Hoebel BG. Hyperphagia and obesity following serotonin depletion by intraventricular $\mathrm{p}$-chlorophenylalanine. Science. 1976:192(4237):382-385.

47. Choi S, DiSilvio B, Fernstrom MH, Fernstrom JD. Meal ingestion, amino acids and brain neurotransmitters: Effects of dietary protein source on serotonin and catecholamine synthesis rates. Physiol Behav. 2009; 98(1-2):156-162.

48. Pedroso JAB, Zampieri TT, Donato J. Reviewing the effects of I-leucine supplementation in the regulation of food intake, energy balance, and glucose homeostasis. Nutrients. 2015;7(5):3914-3937.

49. Su Y, Lam TKT, He W, et al. Hypothalamic leucine metabolism regulates liver glucose production. Diabetes. 2012;61(1):85-93.

50. Arrieta-Cruz I, Gutiérrez-Juárez R. The role of circulating amino acids in the hypothalamic regulation of liver glucose metabolism. Adv Nutr. 2016;7(4):790S-797S

51. Arrieta-Cruz I, Su Y, Gutiérrez-Juárez R. Suppression of endogenous glucose production by isoleucine and valine and impact of diet composition. Nutrients. 2016;8(2):79. 
52. Haufe S, Engeli S, Kaminski J, et al. Branched-chain amino acid catabolism rather than amino acids plasma concentrations is associated with diet-induced changes in insulin resistance in overweight to obese individuals. Nutr Metab Cardiovasc Dis. 2017;27(10):858-864.

53. Jang C, Oh SF, Wada S, et al. A branched-chain amino acid metabolite drives vascular fatty acid transport and causes insulin resistance. Nat Med. 2016;22(4):421-426.

54. Newgard CB, An J, Bain JR, et al. A branched-chain amino acid-related metabolic signature that differentiates obese and lean humans and contributes to insulin resistance. Cell Metab. 2009;9(4):311-326.

55. Mardinoglu A, Gogg S, Lotta LA, et al. Elevated plasma levels of 3-hydroxyisobutyric acid are associated with incident type 2 diabetes. EBioMedicine. 2018;27:151-155.

56. Asghari G, Farhadnejad H, Teymoori F, Mirmiran P, Tohidi M, Azizi F. High dietary intake of branched-chain amino acids is associated with an increased risk of insulin resistance in adults. J Diabetes. 2018;10(5): 357-364.
57. Zheng Y, LiY, Qi Q, et al. Cumulative consumption of branched-chain amino acids and incidence of type 2 diabetes. Int J Epidemiol. 2016; 45(5):1482-1492.

58. Mignone LE, Wu T, Horowitz M, Rayner CK. Whey protein: The 'whey' forward for treatment of type 2 diabetes? World J Diabetes. 2015;6(14): 1274-1284.

59. Yoshizawa F. New therapeutic strategy for amino acid medicine: Notable functions of branched chain amino acids as biological regulators. J Pharmacol Sci. 2012;118(2):149-155.

60. Takamura T, Takeshita Y, Kaneko S. Branched chain amino acids supplementation and glycemic control. In: Rajendram R, Preedy V, Patel V, eds. Branched Chain Amino Acids in Clinical Nutrition. Nutrition and Health. New York, NY: Humana Press; 2015:229-238.

61. Cornelis MC, Hu FB. Systems Epidemiology: A new direction in nutrition and metabolic disease research. Curr Nutr Rep. 2013;2(4):225-235. 